\title{
Essais
}

ESSAIS

Revue interdisciplinaire d'Humanités

$2 \mid 2012$

Aux marges de l'humain

\section{Sujet et voix : questions à la littérature moderne}

\section{Dominique Rabaté}

\section{(2) OpenEdition}

Journals

Édition électronique

URL : https://journals.openedition.org/essais/10520

DOI : 10.4000/essais. 10520

ISSN : 2276-0970

\section{Éditeur}

École doctorale Montaigne Humanités

\section{Édition imprimée}

Date de publication : 15 novembre 2012

Pagination : 93-103

ISBN : 978-2-86781-857-8

ISSN : $2417-4211$

Référence électronique

Dominique Rabaté, "Sujet et voix : questions à la littérature moderne», Essais [En ligne], 2 | 2012, mis en ligne le 17 janvier 2022, consulté le 20 janvier 2022. URL : http://journals.openedition.org/essais/ 10520 ; DOI : https://doi.org/10.4000/essais.10520 
Dominique Rabaté

Université Denis-Diderot Paris 7, EA 4410, CERILAC

\section{N.D.L.R.}

Conférence prononcée à l'université Bordeaux 3 dans le cadre des Horizons de la recherche le mardi 17 janvier 2012. 


\section{Sujet et voix : questions à la littérature moderne}

\section{Dominique Rabaté}

C'est vraiment un grand plaisir pour moi d'être invité ce soir dans une université où j’ai longtemps enseigné, où j'ai dirigé une équipe de recherche, le Centre de recherche sur les modernités littéraires, et où j'ai été directeur de l'École Doctorale, au côté d'Alain Bresson d'abord, puis co-directeur avec mes amis François Bart et Patrick Henriet. C'est dire que je me sens encore un peu chez moi, dans ce cadre des Horizons de la recherche dont j'ai initié, il y a longtemps, la formule qui dure encore et dont j'ai suivi régulièrement les cycles avec un grand bonheur, celui d'apprendre d'autres disciplines, de voir comment s'élaborent pour chaque chercheur les objets de travail. Je vais donc me livrer à un exercice que j'ai souvent demandé à d'autres, en remerciant la direction actuelle de l'École Doctorale, Isabelle Poulin, Pierre Beylot et Sandro Landi de leur confiance.

Ces remerciements inauguraux peuvent sembler un simple lieu rhétorique un peu vide. Je voudrais pourtant souligner qu'ils entrent déjà pour moi dans quelque chose de capital dans la vie d'un chercheur: une certaine économie du singulier et du collectif où joue la nécessité d'institutionnaliser des lieux de travail. Car il va d'un certain équilibre entre le personnel - et dans les SHS cette part est essentielle - et l'impersonnel, équilibre ou dialectique qui prend dans mes réflexions récentes une part de plus en plus importante, et qui devient même à mes yeux l'un des traits définitoires de l'expérience littéraire ou esthétique.

Raconter l'histoire de son itinéraire de recherche, c'est sans doute donner la forme réordonnée d'un récit à ce qu'un trajet réel a pu avoir de plus hésitant et de plus obscur. Mais peut-être s'agit-il aussi, dans la vie intellectuelle, de penser soi-même la logique d'un parcours pour le revendiquer comme sien et pour ainsi dire en devenir à part entière l'auteur, le signataire résolu. On pourrait dire que c'est d'ailleurs ce qui se passe au moment où l'on soutient sa thèse, et où l'on devient le défenseur de ses propres idées.

Je commencerai donc par une anecdote: il y a très longtemps une amie m’avait demandé pourquoi j'aimais La Chute de Camus. Je me souviens que j'avais été très étonné de ne pas pouvoir répondre à cette simple demande, frappé de mon incapacité à raconter l'histoire du livre - dont elle semblait penser que 
c'était elle qui détenait le pouvoir d'intéresser ou non un lecteur. Pour moi, le récit de Camus résidait essentiellement dans son très singulier effet de voix et de présence, oblitérant toute intrigue. D'une certaine façon, j'ai commencé à travailler pour m'expliquer cet étrange pouvoir de la voix dans certains textes, pour réussir - mieux que je n'avais alors su faire - à caractériser les modalités de fascination que ces textes exerçaient sur moi. Cet effort d'élucidation pour moi (et peut-être toute recherche est-elle d'abord cela) devait ainsi prendre la voie d'une description théorique de ce qui se passait dans ces textes.

Ainsi très tôt en maîtrise (comme on disait alors) j'ai choisi de travailler sur Le Bavard de Louis-René des Forêts, ce livre publié en 1946 et qui se présente comme un exercice sarcastique d'autodestruction. Récit d'un homme qui s'afflige de sa propension à parler pour ne rien dire, qui échoue à raconter ses différentes crises et à se constituer par son discours autobiographique biaisé comme sujet entier. L'analyse de ce texte m'amenait à approfondir la logique de l'énonciation qui était à l'œuvre. Répondre à la question "Qui parle?» dans un texte littéraire, c'est en effet constater que les marques subjectives sont coupées de toute vérification empirique. C'est au-delà des outils féconds de la linguistique de l'énonciation, s'apercevoir que le sujet qui s'exprime là est un sujet clivé et pluriel, un sujet scindé radicalement en deux, que la psychanalyse peut aider à penser dans ses paradoxes constitutifs.

D'emblée ce sont donc les rapports entre sujet et voix (à ne pas trop vite confondre) qui ont été au centre de mes préoccupations, avec l'idée d'une inscription dynamique et mobile du sujet dans les énoncés où il se laisse saisir. En ce sens, que j'ai précisé dans ma thèse, mon objectif était immédiatement de contester le schéma trop typologique de la narratologie, loin de tout système structural ou structuraliste. Je m'attachai au contraire à un processus où le sujet de la narration est instable, et dont la place incertaine est l'enjeu dramatique du texte. De l'énoncé premier du Bavard (" Je me regarde souvent dans la glace ") au congé ironique et grinçant qu'il donne à son destinataire, le narrateur du récit de des Forêts ne cesse de changer de position, de se retourner contre et vers lui-même, d'anticiper ce qu'autrui peut penser de lui.

Ce premier travail, prolongé dans le doctorat en élargissant à la fois à toute l'œuvre de des Forêts mais aussi à un pan de récits de la modernité, m’a aussi amené à réfléchir à l'articulation entre le commentaire des textes (attentif à leur dynamique la plus fine) et la théorie plus générale. Pour moi, le texte doit moins se plier à une grille pré-établie que conduire à la contester, à en compliquer la figure. Je crois que j'ai eu très tôt ce désir de mettre en difficulté la théorie littéraire (qui existait effectivement sous ce nom au début des années 1980), de la mettre à l'épreuve de textes moins canoniques. Avec le temps, je m'aperçois aussi que je prends de plus en plus de plaisir à écrire des lectures, à rendre compte de l'effet absolument singulier qu'un livre fait sur 
moi (postulant que cet effet sur moi est en vérité l'effet que tout lecteur peut sentir aussi). C'est ce qui m'a conduit à analyser pour eux-mêmes des récits de Conrad (Heart of Darkness) et de Woolf (To the Lighthouse), ou des livres de Gérard Macé, Patrick Modiano ou Jean-Loup Trassard. Je n’ai pas perdu ce goût de l'explication qui avait guidé mes premiers pas académiques sur la piste du Bavard, et l'étrange nécessité que j'éprouve à m'expliquer d'abord à moimême l'effet particulier d'une œuvre, son accent unique.

La navette nécessaire entre commentaire et théorie (qui se nourrissent l'un l'autre) que je revendique maintenant me conduit à une autre remarque, à mes yeux importante: il ne s'agit pas d'éprouver le schéma général par des textes singuliers pour en améliorer la description, mais plutôt pour penser la relation entre une forme et une force (force que la narratologie sous-estime en la typologisant). On se souvient que c'est ainsi que Derrida répondait au beau livre de Jean Rousset, Forme et signification. Force autant que forme, forme qui se laisse déborder mais qui permet de donner figure à une dynamique qui lui échappe pourtant.

Être attentif aux jeux des voix dans un récit, c'est certainement commencer par entendre cette disjonction possible et cette dialectique complexe entre forme et force. Le Bavard, mais aussi La Chute, comme Les Notes du sous-sol de Dostoïevski, mais encore Beckett, Céline, Bernhard: il s'agissait dès le départ pour moi pas seulement d'œuvres isolées mais d'un mouvement, d'un courant de la modernité. Et ce mouvement, c'est justement celui de l'émancipation de la voix. D'une voix unique mais plurielle, selon la possibilité que la langue française offre de ne jamais savoir si le mot " voix » est au singulier ou au pluriel.

En travaillant sur un ensemble de textes dont le point commun est le passage au premier plan d'une voix narrative (fictive) qui occupe tout l'espace discursif ou qui peut le partager avec d'autres parleurs de monologues croisés (comme chez Faulkner), on doit analyser un infléchissement du roman moderne. À partir de des Forêts ou de Beckett, c'est l'étiquette même de roman qui se trouve contestée, qui s'avère inadéquate, et à la suite de Blanchot j'ai donc proposé de penser le "récit" comme une notion opératoire pour tout le $\mathrm{Xx}^{\mathrm{e}}$ siècle, de Valéry et Gide à quantité de textes contemporains. Récit que je situe volontairement comme "l'ombre du roman ", dans l'ombre du roman, puisque l'effort que le narrateur déploie pour coïncider avec sa propre source énonciative éloigne le récit des développements du roman. Cet effort pourrait se décrire comme la tentative de réduire, de combler ou d'annuler l'écart - par essence incomblable - qui sépare et distingue sujet de l'énonciation et sujet de l'énoncé. Je suis revenu dans Le Chaudron fêlé sur cette notion d'écart qui a pris progressivement une dimension plus théorique pour moi.

De la lecture du Bavard, en croisant Beckett, Blanchot ou Camus, c'est ainsi un pan entier de la littérature $\mathrm{du} \mathrm{xx}^{\mathrm{e}}$ siècle que j'ai essayé de décrire 
en proposant de le nommer par le but - impossible mais paradoxalement productif - qu'il se fixe: celui d'un épuisement tangentiel, toujours différé, d'un échec fécond si l'on veut. C'est pour cela que j'ai doublé le livre sur des Forêts d'un autre, concomitant, intitulé Vers une littérature de l'épuisement.

Je crois que le premier terrain qu'un chercheur se fixe (sans pouvoir évidemment en mesurer alors tous les enjeux et toutes les conséquences) est très significatif. C'est là que se constitue une sorte d'observatoire privilégié qui commandera ensuite le jeu des perspectives futures. En ce sens, l'élection de des Forêts reste pour moi un geste inaugural aux multiples effets, dont je voudrais décrire certains maintenant.

L'œuvre de des Forêts offre une diversité générique quej'ai voulu comprendre; premier roman à l'américaine pour Les Mendiants, récit destructeur, recueil de nouvelles, poèmes, autobiographie à la troisième personne enfin avec Ostinato. D'emblée je ne pouvais me spécialiser dans un aucun genre (même au sens large) et m'engageais à devoir penser les effets d'énonciation spécifiques au choix de formes si dissemblables - alors même que les contenus thématiques restent les mêmes de façon obsédante.

Choisir des Forêts, c'était aussi refuser de céder aux effets mimétiques de certaines (mais rares) études qui avaient été déjà consacrées à cet auteur (peu travaillé quand j'ai commencé à m’intéresser à lui, et quasiment inconnu de l'université). La magnifique postface au Bavard de Blanchot, "La Parole vaine » semblait condamner à faire du mauvais pastiche, ou à s'enfermer dans les vertiges de l'œuvre. Contre ce risque, j'ai choisi de travailler aussi d'autres œuvres, d'ouvrir à la question de l'épuisement une œuvre qui trouvait aussi là sa nécessité et son inscription dans un courant fondamental. C'est une façon de se ménager une marge de manœuvre, un espace de dégagement qui me paraît essentiel. Beaucoup d'études ou de thèses sur des auteurs contemporains (souvent excellents théoriciens de leur propre écriture) sont prises dans un mimétisme agaçant, dans l'éloge d'une œuvre qu'elles ne font que répéter, sans en éclairer la nécessité pour nous.

Mon choix initial me conduisait aussi (et je m'en suis aperçu progressivement avec plus de clarté) à prendre ce que j’ai nommé « le risque du contemporain ». Rendre compte d'un texte contemporain (et ceux de Louis-René des Forêts l'étaient au moment où j'ai commencé à travailler sur eux), c'est forcément devoir rendre compte de la nécessité de l'œuvre parce qu'elle n'a pas encore reçu de reconnaissance indiscutable. C'est d'ailleurs œuvrer à cette reconnaissance, et je dois avouer que je suis fier d'avoir contribué avec d'autres (Bernard Pingaud, Jean Roudaut notamment) à mettre mieux en lumière une œuvre qui est mieux appréciée aujourd'hui. Cette obligation d'expliquer les raisons du choix d'un commentaire me semble précieuse, parce qu'elle oblige à expliciter le geste critique que l'on veut accomplir. Pareille injonction manque 
pour des travaux sur des auteurs consacrés, comme si cela allait de soi de se consacrer à Racine, Voltaire ou Proust, alors qu'il conviendrait plutôt, selon moi, d'expliquer et expliciter la nécessité de ce retour, la pertinence de cette relecture. En un mot, il me semble qu'un critique littéraire doit toujours situer sa propre parole et, autant que possible, en apprécier les effets.

Je voudrais confier une autre anecdote: la nécessité dont je parle, je l'ai aussi éprouvée à un autre niveau. Lorsque j'ai commencé ma thèse de doctorat ("nouveau régime» alors comme on la nommait pour la différencier de l'ancienne Thèse d'État), il y a eu un moment de flottement administratif pour savoir si je devais m'inscrire en DEA ou si je pouvais, comme agrégé, attaquer directement en thèse. Mais comme j'étais parti aux États-Unis comme lecteur, je me trouvais un peu en difficulté pour faire valider là-bas des cours. Si je mentionne ce court moment d'hésitation, c'est parce que je crois qu'il m'a été en vérité très utile: confronté à la possibilité de ne pas poursuivre de façon rectiligne mon parcours de bon élève, je me suis dit intérieurement que le travail que je comptais commencer devait se faire, dans quelque cadre que ce fût. L'interrogation que je portais sur les textes de des Forêts devenait ainsi un engagement intime, qu'il me fallait un peu mystérieusement résoudre ou mener à bien en dehors même de tout cadre institutionnel. Si finalement l'affaire s'est réglée au mieux, j'en ai gardé la conviction - qui parfois manque au thésard - que la recherche que j'entreprenais n'était pas seulement académique mais qu'elle impliquait pour moi des choix de pensée, de travail et de vie.

Je profite de ce rappel pour dire aussi combien m'a été précieux ce séjour de trois ans aux États-Unis, suivi de deux ans comme coopérant en Norvège, et comment cette ouverture sur d'autres manières d'enseigner et de travailler a changé mon point de vue. Ce décentrement du regard par rapport à nos habitudes franco-françaises me semble avoir compté, en me donnant une autre forme de liberté et d'autonomie dont je fais donc ici l'éloge, et je vous encourage donc autant que possible à profiter de toutes les occasions et toutes les chances de partir à l'étranger!

Je me souviens aussi que je trouvais assez agréable de n'avoir à emporter que cinq ou six livres pour posséder l'intégralité des livres de des Forêts, puisque j'ai fait ma thèse en partie comme lecteur à l'université aux États-Unis et en Norvège. Pas besoin de malle trop pleine! Et une certaine forme de liberté critique puisque peu avait encore été écrit.

Pour un étudiant qui avait été, comme moi, élevé au biberon structuraliste de la séparation catégorique entre l'écrivain et la personne réelle, le fait de travailler sur un écrivain vivant, de le rencontrer au terme de l'année de maîtrise et même d'en devenir proche, cela a été une expérience importante. Non pas tant pour demander à l'écrivain de valider des analyses que parce que j'avais le sentiment que ce que j'écrivais sur lui, l'auteur pouvait le lire, et que cela 
dictait donc une manière d'écrire prenant en compte le souci de ce destinataire particulier. Une manière de l'engager dans certaines voies, de répondre comme lecteur à la sollicitation forte de ses textes, de rester fidèlement à son écoute.

Pour revenir à des questions plus théoriques, les récits que j'ai mis au centre de mes premiers travaux impliquaient de voir dans le sujet qui parle en première personne, même si c'est souvent de façon anonyme, l'agent d'une recherche de soimême, par le langage. Ou pour le dire autrement, de penser le sujet dans les effets de son langage comme s'ils faisaient retour sur celui qui en est pourtant l'origine. Insister sur les effets, c'est reprendre sciemment le titre d'une collection où Derrida a publié certains de ses livres, "La Philosophie en effet » et souligner l'influence majeure de la pensée de ce philosophe sur toutes mes analyses, notamment par sa critique magistrale du phonocentrisme. De Derrida, je retenais essentiellement la conviction de l'impossibilité d'une pleine et entière présence à soi du sujet parlant. Conviction anti-métaphysique qu'il me semble que la littérature met en pratique pour ainsi dire naturellement ou obligatoirement. Mais la psychanalyse peut aussi délivrer la même leçon, en interrogeant un impensé radical que la phénoménologie ne saurait jamais atteindre.

Sans entrer ici dans des arguments trop techniques ou trop théoriques, la description de ces récits particuliers m'amenait ainsi à prendre position dans le champ des théories du sujet, cette grande affaire littéraire et philosophique $\mathrm{du} \mathrm{Xx}^{e}$ siècle, certainement. Qu'il y ait du sujet, qu'il se traduise ou trahisse en effets, cela doit être rappelé contre toute neutralisation subjective (et en ce sens, je ne souscris pas à tout ce que Blanchot avance quant au neutre). Mais il est impossible de maintenir une représentation unifiée ou même unitaire d'un sujet qu'il faut plutôt penser, grâce à la littérature, comme pluralisé et mobile, jouant sur plusieurs scènes en même temps. Pas de mort du sujet donc, mais la reconnaissance de la part subjective qui joue avec quelque chose d'a-subjectif ou de trans-subjectif en chaque énonciation. Ce que j'ai appelé tout à l'heure la négociation du personnel et de l'impersonnel autour de laquelle je réfléchis à la fin de mon dernier livre, Le Roman et le sens de la vie.

Mon travail de thèse avait ainsi deux orientations: celle de l'étude encore inédite dans l'université d'une ouvre révélatrice (et qu'il fallait décrire comme telle), et un pan plus théorique sur l'inscription du sujet comme voix dans le texte littéraire, sur ce que j'ai qualifié de "récit ». J'ai eu la chance immense de pouvoir très vite publier chez Corti deux livres qui ont paru en même temps et qui formaient donc les deux volets de mon enquête. Je tiens ici à dire toute la reconnaissance que je dois à cet éditeur fidèle et combien je suis heureux de continuer à publier régulièrement chez lui. C'est d'une certaine façon l'un des premiers lecteurs auxquels je m'adresse quand je lui confie un nouveau manuscrit.

Ce premier et double chantier ouvrait aussi en fait bien d'autres perspectives qui se sont retrouvées logiquement impliquées dans mes questionnements initiaux. 
Sujet et voix : questions à la littérature moderne

D'abord du côté de la littérature contemporaine que je pouvais lire comme le prolongement de ce mouvement de l'épuisement, notamment l'œuvre de Pascal Quignard. Mais aussi l'obligation de revenir sur bien des notions touchées par Maurice Blanchot. Ou encore l'intégration de l'œuvre de Céline, à laquelle je n'avais pas touché dans ma thèse, dans la réflexion sur la voix dans la littérature moderne. Chemin qui m’a mené à élargir le spectre des œuvres commentées, vers Louis Guilloux, mais aussi plus près de nous Georges Perec, Emmanuel Carrère, Marie NDiaye ou Jean-Benoît Puech. Je veux dire par là que j'avais pris le goût de maintenir éveillée la curiosité pour les livres nouveaux, pour le surgissement de nouvelles problématiques littéraires (ce qui implique aussi de ne pas se laisser intimider par la fausse nouveauté d'œuvres qui proposent plutôt de la fausse monnaie).

De façon plus directement théorique, la conclusion de Vers une littérature de l'épuisement, m'engageait aussi vers un travail de description et de mise en ordre de ce que j'appelle maintenant des "modalités d'énonciation littéraire " conçues comme des sortes d'archi-genres, dans une perspective plus dynamique que typologique. À côté du récit, il me fallait faire place à la modalité poétique. Et je m'y suis ainsi attelé avec des travaux personnels et collectifs sur le «sujet lyrique ". Et aussi revenir à une meilleure définition (ou compréhension) du roman moderne, comme forme d'expression de la conscience solitaire.

Le parcours d'une recherche est aussi profondément lié à des rencontres, à des amitiés, à des envies de travailler avec d'autres. Et je suis peut-être plus que certains très sensible à cette dynamique qui naît de projets communs, de partages de savoirs et de points de vue. J'ai eu l'immense chance en arrivant en 1991 à Bordeaux 3 de trouver l'équipe "Modernités " qu'y dirigeait Yves Vadé, dont j'ai pris la succession quand il est parti à la retraite. Nous avons ainsi discuté ensemble, réfléchi à des thématiques communes, initié des colloques sur le sujet lyrique, sur l'instant. La générosité de son accueil, la confiance qu'il m’a témoignée ont été tout à fait déterminantes pour moi.

Je voudrais donner un exemple du genre de travaux collectifs que j'ai dirigés pour montrer comment la recherche personnelle peut s'articuler à celle des autres. J'ai proposé comme séminaire de Modernités un travail de deux années intitulé "L'Invention du solitaire ". Je cherchais en effet à prolonger des réflexions sur le monologue, sur la position de solitaire de l'écrivain moderne, notamment dans la conclusion de Vers une littérature de l'épuisement que j'avais appelé selon le mot de Nietzsche "Le jugement du solitaire ». Mon idée était en effet que l'invention de la littérature (à son sens moderne) est le fait du solitaire, de Rousseau à Flaubert, de Baudelaire à Kafka, de Pessoa à Beckett. Mais aussi que c'est peut-être la littérature qui invente les figures nouvelles de la solitude moderne. Pour mener une telle enquête, qui intéresse la littérature européenne ou mondiale, il faut repartir de Rousseau - qui en forme l'indispensable strate archéologique - et 
explorer plus de deux siècles d'écriture (où l'autobiographie et la pratique du journal intime ont une place stratégique). J'ai donc voulu demander à d'autres chercheurs, à d'autres spécialistes d'enrichir mon corpus et mon interrogation pour produire un ouvrage véritablement collectif sur un sujet qu'il ne me semble pas possible d'épuiser seul (comme cela avait pu être l'idéal académique de l'ancienne thèse d'État).

Cette expérience de travail collectif (et celle de la direction d'une équipe d'une trentaine de chercheurs) a déplacé ma façon de travailler, en me rendant de plus en plus absurde le découpage si rigide en siècles qui règne dans l'université française. Il me paraît bien plus profitable de mener les enquêtes critiques sur l'échelle plus vaste de la modernité (depuis Baudelaire), ou même souvent - comme dans le cas de la question du solitaire - de repartir de la fin du XviII ${ }^{\mathrm{e}}$ siècle. Et de ne pas l'enfermer arbitrairement dans la seule littérature française, quand ce type de questions se pose évidemment et au moins à la littérature européenne tout entière. Quand on étudie comme nous l'avons fait au sein de Modernités le secret, le deuil ou plus récemment le nihilisme, cela n'a aucun sens de réduire le questionnement à une littérature nationale. Et la comparaison des contextes nationaux, des effets de langues spécifiques permet au contraire de creuser et d'enrichir l'analyse.

Car, à travers ces différentes enquêtes, où les notions jouent comme des véritables "personnages conceptuels " au sens de Deleuze, il s'agit d'analyser les manières dont la littérature, avec d'autres arts, reflète et modélise les transformations des façons d'être un sujet. Mais ce sujet n'est pas directement donné; il agit comme une question. Pour le dire en d'autres termes voisins, il faut envisager les modes de subjectivation de la modernité, en imaginant pour chaque configuration des cartographies différentes, sans oublier que tout mode de subjectivation implique logiquement aussi des modalités particulières de désubjectivation dans des "dispositifs" qu'il faut décrire de manière globale. Je reprends ici le terme de dispositif aux analyses de Giorgio Agamben relisant Michel Foucault.

C'est dans cette direction très générale que s'orientent maintenant certains de mes travaux. J'essaie par exemple de voir quelles figures originales prend aujourd'hui le motif de la disparition dans le roman contemporain, où il me semble que ce motif traduit à la fois un désir de disparaître pour se soustraire à la norme sociale et au contrôle, mais aussi la conjuration de la peur d'être simplement effacé comme sujet individuel ou collectif. Je retrouve dans cette nouvelle enquête l'importance d'un fonctionnement pour le moins paradoxal et ambivalent, dans lequel le même thème a deux significations contradictoires.

$\mathrm{Ou}$ encore, c'est le processus même d'identification qui me retient depuis quelque temps. Je voudrais l'analyser comme mouvement dynamique où la capacité à s'identifier ne se résume pas à l'obtention d'une identité fixée. Car l'identification présuppose aussi bien la coïncidence d'une certaine 
désidentification qui la rend, en vérité, possible. Là encore les enseignements de la littérature et de la psychanalyse peuvent se croiser de façon féconde. C'est continuer à souligner l'articulation très particulière du plus personnel (qui implique de soi l'engagement le plus résolu) avec une dimension - qui doit être reconnue pour telle - absolument impersonnelle. Car cette dimension impersonnelle relève du rapport au langage et du rapport au temps. Dans ces deux rapports essentiels, un sujet est en relation nécessaire avec ce qu'il lui est pourtant impossible de posséder, quelque chose d'inappropriable, mais qu'il doit cependant énoncer en son nom et vivre et endurer dans sa chair. C'est ce que j'ai tenté de montrer dans les lectures de La Mort d'Ivan Ilitch de Tolstoï et de Promenade au phare de Woolf.

Cette "négociation " capitale, j'ai donc essayé de l'approcher dans Le Roman et le sens de la vie, essai qui est à mes yeux le texte le plus personnel que j'aie écrit, où il me semble que je parviens à dire quelque chose que je cherchais confusément dans mes livres précédents. Wittgenstein fait dans ses Leçons et conversations une très belle remarque, en disant que nous pouvons nous étonner du miracle qu'il y ait un monde. Mais nous pouvons aussi, poursuit-il, nous étonner du miracle qu'il y ait aussi le langage. Sur ce monde comme sur le langage, il n'y aura jamais de point de vue surplombant ou extérieur, ce que le philosophe nomme un " point de vue angélique ». Nous sommes dans ce monde, et dans le langage que nous parlons sans pouvoir nous en abstraire. Mais cet étonnement et ce sentiment de miracle, je crois qu'ils traduisent une expérience de dessaisissement et de ravissement qui fait le fonds de l'expérience esthétique. Dans ce moment d'affirmation de quelque chose d'inespéré et de pourtant banal, nous nous accordons avec des forces impersonnelles que l'art et la littérature ne cessent de nous faire éprouver.

C'est sans doute cette expérience, cent fois renouvelée par les œuvres, qu'il me semble important de décrire et de faire partager, cette expérience que certains textes ou certains films nous font ressentir et que le travail critique et théorique cherche, à son tour, à nommer et caractériser, pour en prolonger la puissance de ravissement.

\section{Bibliographie}

\section{a. Livres personnels:}

Vers une littérature de l'épuisement, José Corti, 1991, réédition en 2003.

Louis-René des Forêts: la voix et le volume, José Corti, deuxième édition revue et augmentée, 2002.

Poétiques de la voix, José Corti "Les Essais », 1999.

Le Chaudron fêlé. Écarts de la littérature, José Corti «Les Essais », 2006.

Le Roman et le sens de la vie, José Corti «Les Essais », 2010. 
Gestes lyriques, José Corti, à paraitre en avril 2013.

Le Roman français depuis 1900, "Que sais-je? ", n 49, PUF, 1998.

Pascal Quignard, Étude de l'œeuvre, Bordas "Écrivains au présent ", 2008.

Marie NDiaye, livre-CD, CulturesFrance et Textuel, 2008.

\section{b. Direction d'ouvrages collectifs:}

Cahier Louis-René des Forêts, avec J.-B. Puech, Le Temps qu'il fait, 1991.

Figures du sujet lyrique, Presses Universitaires de France, 1996.

Modernités 8: Le sujet lyrique en question, avec J. de Sermet et Y. Vadé, Presses Universitaires de Bordeaux, 1996.

Modernités 11: L'instant romanesque, PUB, 1998.

Modernités 14: Dire le secret, PUB, 2001.

Modernités 15:Écritures du ressassement, avec É. Benoit, M. Braud, J.-P. Moussaron et I. Poulin, Presses Universitaires de Bordeaux, 2001.

Critique n 668-669: Louis-René des Forêts, Minuit, 2003.

Modernités 19: L'Invention du solitaire, Presses Universitaires de Bordeaux, 2003.

Poésie et autobiographie, avec E. Audinet, cipM et farrago, 2004.

Modernités 21: Deuil et littérature, avec P. Glaudes, Presses Universitaires de Bordeaux, 2005.

Modernités 25: L'Art et la question de la valeur, Presses Universitaires de Bordeaux, 2007.

Littérature et sociologie, avec Ph. Baudorre et D. Viart, Presses Universitaires de Bordeaux, "Sémaphores ", 2007.

Modernités 29: Puissances du mal, avec P. Glaudes, Presses Universitaires de Bordeaux, 2008.

Écritures blanches, avec D. Viart, Publications de l'Université de Saint-Étienne, 2009.

Modernités 31: En quel nom parler?, Presses Universitaires de Bordeaux, 2010. Revue critique de fixxion française contemporaine, revue électronique, avec Pierre Schoentjes, n 1: «Micro/macro », janvier 2010.

Europe $\mathrm{n}^{\circ}$ 976-977: Pascal Quignard, avec Alexandre Gefen, septembre 2010. ELFE $\mathrm{n}^{\circ} 1$ (Revue de la Société de Littérature Française du $\mathrm{xx}^{\mathrm{e}}$ siècle), L'Aventure, avec Didier Alexandre et Nathalie Froloff, Classiques Garnier, octobre 2011.

Samuel Beckett Today/Aujourd'hui n ${ }^{\circ} 23$ : Filiations et connexions. Filiations \& Connecting Lines, avec Sjef Houpermans et Yann Mével, Rodopi, 2011.

Modernités 33: Nihilismes?, avec Éric Benoit, Presses Universitaires de Bordeaux, 2012. 